Bio - grafia. Escritos sobre la Biología y su Enseñanza. ISSN 2027

Edición Extraordinaria. p.p. 295 - 303

Memorias del VIII Encuentro Nacional de Experiencias en Enseñanza de la Biología y la Educación Ambiental. III Congreso Nacional de Investigación en Enseñanza de la Biología.

\title{
AVANCE DE INVESTIGACION: CONSTRUCCION DE CATEGORIAS DE ANALISIS PARA DETERMINAR LA INCLUSION DE LA EDUCACIÓN AMBIENTAL EN LA FORMACIÓN DE PROFESIONALES EN BIOLOGIA.
}

\section{ADVANCE RESEARCH: BUILDING ANALYSIS CATEGORIES FOR DETERMINE THE INCLUSION OF ENVIRONMENTAL EDUCATION IN THE FORMATION OF PROFESSIONALS IN BIOLOGY.}

Pérez Cediel Carlos Andrés ${ }^{1}$, Sánchez Bonell David Andrés²

\section{RESUMEN}

En esta investigación para lograr identificar como se encuentra incluida la Educación Ambiental en el programa de Biología de la Universidad del Tolima, se hace necesario plantear un sistema de categorías que permitan la construcción de instrumentos, interpretación y análisis de documentos y datos, así como la discusión de resultados. Para lograr el objetivo, se plantean tres categorías que engloban los diferentes temas que se deben tener en cuenta en un currículo ambientalizado: (A) concepción de ambiente, (B) tendencia en Educación Ambiental y $(C)$ ambientalización curricular; y cada categoría cuenta con unas subcategorías que entre ellas se entrelazan y construyen la categoría superior y la unión de las categorías permiten diagnosticar el estado de la Educación Ambiental.

Palabras claves: Educación Ambiental, categorías de análisis, currículo, biología.

\section{ABSTRACT}

In this investigation, in order to identify how the Environmental Education is included at University of Tolima biology program, it is necessary to consider a system of categories which allows the construction of instruments, interpretation and analysis of documents and data, as well as the discussion of results. To achieve the goal, there are three categories that encompass the different issues that should be considered in an environmentalized curriculum: (A) Conception of environment, (B) Trends in Environmental Education and (C) Curriculum Greening. Each category has subcategories which are intertwined among them

\footnotetext{
${ }^{1}$ Universidad Militar Nueva Granada, Maestría en Educación, carlosperezcediel@gmail.com

2 Universidad Militar Nueva Granada, Maestría en Educación, dsanchez.david@gmail.com
} 
Bio - grafia. Escritos sobre la Biología y su Enseñanza. ISSN 2027

Edición Extraordinaria. p.p. 295 - 303

Memorias del VIII Encuentro Nacional de Experiencias en Enseñanza de la Biología y la Educación Ambiental. III Congreso Nacional de Investigación en Enseñanza de la Biología.

and at the same time construct a superior category. The union of these categories, allows the researcher to diagnose the state of Environmental Education.

Key words: Environmental Education, categories of analysis, curriculum, biology.

\section{INTRODUCCION}

La educación ambiental juega un papel importante en la sociedad actual, debido a que permite dar explicación a la realidad ecológica de los ecosistemas, da razón sobre la utilización de los recursos y genera las posibilidades de poder utilizar dichos recursos para el beneficio de las poblaciones y además permite que los problemas ambientales no sean vistos únicamente desde el punto de vista biológico, sino que también facilita que se analicen desde un enfoque social, político y económico (Marcote, 2005) (Sauvé, 2004).

En Colombia, dentro de la educación formal, la Educación Ambiental se ha ido introduciendo en los currículos de las instituciones de educación básica y superior; este proceso ha sido sustentado por la Ley 115 de 1994 y la Ley 30 de 1993, las cuales plantean que la educación debe ser el instrumento para la solución de los problemas ambientales e indican que la educación se debe fundamentar en la buena relación de los seres humanos con el ambiente (SINA, 2002).

Con los temas relacionados con la Educación Ambiental, se tiene la concepción que esta solo la realizan los licenciados en ciencias naturales, esto debido a el termino educación que se cree de uso exclusivo de los docentes. Sin embargo, teniendo en cuenta que el estudio del ambiente se lleva a cabo por las ciencias naturales como las sociales, la Educación Ambiental puede abordarse desde diferentes perspectivas, dándole característica de interdisciplinar y transversal (Pedroza, R. \& Argüello F. 2002). Los profesionales abordan el conocimiento del ambiente a partir de su conocimiento disciplinar y por lo tanto se hace necesario determinar, como está incluida la educación ambiental dentro de los currículos universitarios y en el caso de esta investigación, en la formación de los profesionales en biología de la Universidad del Tolima. 
Bio - grafia. Escritos sobre la Biología y su Enseñanza. ISSN 2027

Edición Extraordinaria. p.p. 295 - 303

Memorias del VIII Encuentro Nacional de Experiencias en Enseñanza de la Biología y la Educación Ambiental. III Congreso Nacional de Investigación en Enseñanza de la Biología.

Desde este orden de ideas, se hace necesario el análisis curricular del programa de formación en Biología de la Universidad del Tolima y para lograr este cometido se hace imprescindible la construcción de unas categorías de análisis que soporten este objetivo.

\section{CONSTRUCCION DE CATEGORIAS}

La categorización es una de las características que posee la investigación cualitativa y está basada en la creación y uso de categorías para análisis; y teniendo en cuenta que la categoría es el elemento o dimensión de cada una de las variables que se encuentran bajo estudio y cuya función es la de clasificar o reunir los datos o variables de la investigación (López, 2002). Esto permite establecer la importancia de plantear un sistema de categorías que permita fundamentar los métodos de recolecta y análisis de información y consolidarse como la base de esta investigación.

Para determinar la inclusión de la Educación Ambiental en el currículo del programa de biología se plantearon tres categorías de análisis: Las categorías de análisis se construyeron teniendo en cuenta tres ejes fundamentales para la investigación: ambiente, Educación Ambiental y el currículo en ambiental. Motivo por el cual se plantearon tres categorías de análisis que se denominaron: $A$ ) concepciones de ambiente, B) tendencia en Educación Ambiental y C) ambientalización curricular.

De una forma general, las categorías buscan organizar para el análisis la información que procede de la comunidad universitaria, así como la proveniente de los documentos $(A)$ se pretende identificar las diferentes concepciones que se tiene de ambiente, analizando dichas concepciones a partir de la epistemología del concepto; con (B) se pretende dilucidar la percepción de estudiantes y egresado con respecto a la forma en que el programa de biología trabaja la Educación Ambiental; y por último en (C) se busca identificar de qué manera se encuentra la Educación Ambiental dentro del plan curricular del programa de biología a través de las actividades académicas.

Estas categorías a la vez se dividieron a su vez en subcategorías que permitirán un análisis más apropiado a los objetivos planteados; de esta manera la categoría (A) presenta tres subcategorías, (B) se encuentra representada por cuatro y la categoría (C) por cinco; la división de categorías y subcategorías se visualiza en la tabla1. Además, de cada subcategoría se construyeron unas afirmaciones que sustentan la categoría y las cuales 
Bio - grafia. Escritos sobre la Biología y su Enseñanza. ISSN 2027

Edición Extraordinaria. p.p. 295 - 303

Memorias del VIII Encuentro Nacional de Experiencias en Enseñanza de la Biología y la Educación Ambiental. III Congreso Nacional de Investigación en Enseñanza de la Biología.

fueron la base para la construcción de los instrumento de toma de datos, como lo son: Cuestionario tipo Likert, la entrevista semi-estructurada y el análisis documental.

A continuación se muestran las categorías con sus respectivas subcategorías; para analizar las concepciones de ambiente, es necesario categorizar el término y dividirlo en tres subcategorías, las cuales serán identificadas como: (A1) naturalista, (A2) simbólica y (A3) sistémica. En este punto, se hace necesario especificar que la construcción de la categoría, tuvo como referente las aportaciones de algunos autores. Dichas aportaciones contemplan las concepciones de ambiente desde punto de vistas muy variados, como lo son: concepciones simplistas o biológicas, simbólicas, criticas, sistémicas, contextuales y complejas (Sauvé 2004) (Vidart 1996) (Pedroza, R. \& Argüello F. 2002) (Alvarado \& García 2008). Las subcategorías se explicaran a continuación.

Las concepciones de ambiente pueden girar alrededor de una definición de ambiente que toma como fundamento solamente el componente físico, químico y biótico, identificando al ambiente como un inventario de recursos, este sería un enfoque naturalista o biológico (A1).

Tabla 1. Categorías de análisis y sus respectivas subcategorías

\begin{tabular}{|c|c|c|}
\hline$(A)$ & $\begin{array}{c}\text { (B) TENDENCIA EN } \\
\text { EDUCACION } \\
\text { AMBIENTAL (TEA) }\end{array}$ & $\begin{array}{c}\text { (C) AMBIENTALIZACION } \\
\text { CURRICULAR (AC) }\end{array}$ \\
\hline $\begin{array}{c}\text { A1. El ambiente se } \\
\text { toma como la } \\
\text { interacción de } \\
\text { componentes } \\
\text { geofísicos, químicos y } \\
\text { biológicos, en donde } \\
\text { se desarrolla la vida }\end{array}$ & $\begin{array}{c}\text { B1. Disciplinar. Se estudia } \\
\text { la EA como una disciplina } \\
\text { con un enfoque especifico }\end{array}$ & $\begin{array}{c}\text { C1. Se reconoce la importancia de } \\
\text { la fundamentación en EA }\end{array}$ \\
\hline $\begin{array}{c}\text { A2. El ambiente } \\
\text { además de ser un } \\
\text { conjunto de factores } \\
\text { naturales presenta }\end{array}$ & $\begin{array}{c}\text { B2. Multidisciplinar. La EA } \\
\text { se trabaja teniendo en } \\
\text { cuenta varias disciplinas } \\
\text { para desarrollar un tema en }\end{array}$ & $\begin{array}{c}\text { C2. En el plan de estudios se } \\
\text { encura explicita la enseñanza } \\
\text { de la EA }\end{array}$ \\
\hline
\end{tabular}


Bio - grafia. Escritos sobre la Biología y su Enseñanza. ISSN 2027

Edición Extraordinaria. p.p. 295 - 303

Memorias del VIII Encuentro Nacional de Experiencias en Enseñanza de la Biología y la Educación Ambiental. III Congreso Nacional de Investigación en Enseñanza de la Biología.

\begin{tabular}{|c|c|c|}
\hline un componente social & $\begin{array}{l}\text { común, pero cada disciplina } \\
\text { conservando su enfoque }\end{array}$ & \\
\hline \multirow[t]{3}{*}{$\begin{array}{l}\text { A3. El ambiente se } \\
\text { toma como el sistema } \\
\text { donde convergen las } \\
\text { condiciones físicas y } \\
\text { biológicas con el } \\
\text { componente social, } \\
\text { político y económico }\end{array}$} & $\begin{array}{l}\text { B3. Interdisciplinar. En la } \\
\text { EA existe dialogo e } \\
\text { interacción entre las } \\
\text { disciplinas, buscando punto } \\
\text { de interés y buscando la } \\
\text { solución de una } \\
\text { problemática común }\end{array}$ & $\begin{array}{c}\text { C3. Se incorporan problemáticas } \\
\text { ambientales en los planes de } \\
\text { estudio }\end{array}$ \\
\hline & $\begin{array}{l}\text { B4. Transdisciplinar. La EA } \\
\text { integra el conocimiento a } \\
\text { través de } \\
\text { interdisciplinariedad } \\
\text { eliminando por completo las } \\
\text { fronteras entre las } \\
\text { disciplinas. }\end{array}$ & $\begin{array}{l}\text { C4. En la metodología de trabajo } \\
\text { en el aula se observa la influencia } \\
\text { de la educación ambiental }\end{array}$ \\
\hline & & $\begin{array}{c}\text { C5. Se desarrolla investigación en } \\
\text { el área de la EA }\end{array}$ \\
\hline
\end{tabular}

Fuente: autores

Cuando se tiene en cuenta, además del componente biológico para la definición de ambiente, un componente social y de las relaciones de las unidades de la sociedad y la formación de comunidades, se habla de un enfoque simbólico (A2). Esta subcategoría se encuentra apoyada en los planteamientos de Sauvé (2004).

Cuando se observa al ambiente teniendo en cuenta la interacción de las características físicas y biológicas para la formación de ecosistemas, en los cuales se observa un flujo constante de energía por estas relaciones y cuando en un contexto determinado, se hace interaccionar la parte física, biótica, económica y sociocultural en la explicación del ambiente; para su definición se le considera como un sistema, el cual funciona como un engranaje con muchas interacciones (Vidart, 1996) (Pedroza, R. \& Argüello F. 2002) 
Bio - grafia. Escritos sobre la Biología y su Enseñanza. ISSN 2027

Edición Extraordinaria. p.p. 295 - 303

Memorias del VIII Encuentro Nacional de Experiencias en Enseñanza de la Biología y la Educación Ambiental. III Congreso Nacional de Investigación en Enseñanza de la Biología.

(Alvarado \& García 2008). De esta manera se da una definición global del ambiente se habla de un enfoque sistémico (A3).

Para la categoría (B), con la que se pretende: identificar el enfoque con el cual se trabaja la Educación Ambiental en la Universidad del Tolima; se tomaron como referencia, los enfoques planteados por Covas (2010), Mora (2011), Boada (2005) Sauvé (2004), Pedroza y Argüello (2002), Llorente y Gutiérrez (2006), Gonzales (1996) y Pujol (2003). Y a partir de estos planteamientos, se construyen cuatro subcategorías en la cual se dan relevancia en su clasificación al trabajo disciplinar de la Educación Ambiental y al hacer eso, abren las puertas a que diferentes áreas del conocimiento trabajen a el ambiente como eje. Además, al dar el nombre de disciplina no se hace necesaria la división de la categoría entre ciencias naturales y ciencias sociales como los agentes de acción; y esto debido a que un enfoque disciplinar es más completo y diverso. Y estas cuatro subcategorías, las cuales engloban las diferentes corrientes o enfoque en los cuales se desarrolla o trabaja la Educación Ambiental; y se plantean de la siguiente manera: (B1) disciplinar, (B2) multidisciplinar, (B3) interdisciplinar y (B4) Transdisciplinar.

En la subcategoría (B1) Disciplinar, en este enfoque, se toma a la Educación Ambiental como una disciplina independiente, como el tema de estudio independiente y referente a una sola disciplina, en otras palabras, un enfoque específico; con esto se puede decir que como disciplina posee las herramientas necesarias para realizar investigación desde ella y que posee su propio marco conceptual y epistemológico.

En la subcategoría (B2) el enfoque es Multidisciplinar, la Educación Ambiental se trabaja teniendo en cuenta varias disciplinas para desarrollar un tema en común, pero cada disciplina conservando su área de conocimiento; por lo que puede extraer conceptos y métodos de varias disciplinas pero cada disciplina conserva su marco conceptual.

Con (B3), en esta subcategoría, existe dialogo e interacción entre varias disciplinas, buscando un punto de interés y la solución de un problema común. Las disciplinas que se encuentran en dialogo, combinan sus conocimientos y metodologías para alcanzar su cometido; en este punto se habla de un enfoque interdisciplinar.

Al decir que la Educación Ambiental es Transdisciplinar (B4) los conocimientos de varias disciplinas se integran a través de interdisciplinariedad eliminando por completo las fronteras entre las disciplinas; la Educación Ambiental traspasa, atraviesa los diferentes 
Bio - grafia. Escritos sobre la Biología y su Enseñanza. ISSN 2027

Edición Extraordinaria. p.p. 295 - 303

Memorias del VIII Encuentro Nacional de Experiencias en Enseñanza de la Biología y la Educación Ambiental. III Congreso Nacional de Investigación en Enseñanza de la Biología.

saberes convirtiéndose en eje integrador a través de la sinergia disciplinar, la educación ambiental es el eje de sinergia de las disciplinas.

Para la categoría de Ambientalización curricular $(C)$ se pretende identificar el grado en el cual se encuentra inmersa dentro del currículo la Educación Ambiental; la universidad adquiere una responsabilidad social y debe abordar $e$ introducir en sus currículos la educación ambiental (Gómez \& Botero 2012) (Mora 2011). Teniendo en cuenta esta justificación, se dividió esta categoría en cinco subcategorías, las cuales tiene en cuenta las diez características definidas por la Red ACES (Programa de Ambientalización Curricular de los Estudios Superiores) para determinar el grado de ambientalización curricular (Calabuig 2004, pág 255-257), estas características se fusionaron según la pertinencia y similitud en el objetivo a alcanzar.

Para la primera subcategoría (C1). Se reconoce la importancia de la fundamentación en Educación Ambiental, se busca identificar: se sabe que es Educación Ambiental, se reconocen sus fundamentos, hay una postura clara y se conoce la importancia de ella en la formación de profesionales. Con la subcategoría (C2), se pretende identificar si en el plan de estudios se encuentra explicita la enseñanza de la Educación Ambiental y de la misma manera en la subcategoría (C3) se espera medir la incorporación de problemáticas ambientales en los planes de estudio del programa académico; en la subcategoría (C4) se plantea como se observa la influencia de la Educación Ambiental en la metodología de trabajo en el aula y por último en (C5) se busca identificar si dentro del programa académico se realiza investigación en el área de la Educación Ambiental.

A partir de la construcción de estas categorías de análisis, se cimientan las bases para el desarrollo de la investigación y proporciona un método eficaz para la clasificación y análisis de todos los componentes curriculares del programa de Biología de la Universidad del Tolima.

\section{REFERENCIAS}

Alvarado, L. J., \& García, M. (2008). Características más relevantes del paradigma socio-crítico: su aplicación en investigaciones de educación ambiental y de enseñanza de las ciencias realizadas en el Doctorado de Educación del Instituto Pedagógico de Caracas. Sapiens: Revista Universitaria de Investigación, 9(2), 187-202.

Boada, D., \& Escalona, J. (2005). Enseñanza de la educación ambiental en el ámbito mundial. Educere, 9(30), 317-322. 
Bio - grafia. Escritos sobre la Biología y su Enseñanza. ISSN 2027

Edición Extraordinaria. p.p. 295 - 303

Memorias del VIII Encuentro Nacional de Experiencias en Enseñanza de la Biología y la Educación Ambiental. III Congreso Nacional de Investigación en Enseñanza de la Biología.

Calabuig, T. C. (2004). Diagnóstico del grado de ambientalización de la materia Didáctica de las Matemáticas de la Facultad de Educación y Psicología de la UdG. En: Nuevas tendencias en investigaciones en Educación Ambiental, serie educación ambiental. 249, Madrid

Covas. A. O.(2010). EDUCACIÓN AMBIENTAL A PARTIR DE TRES ENFOQUES: COMUNITARIO, SISTÉMICO E INTERDISCIPLINARIO. recuperado el 17 noviembre de 2013, en:http://www.ambiente.gov.ar/infotecaea/descargas/covas01.pdf

Gómez, C., \& Botero, C. M. (2012). La ambientalización de la educación superior: estudio de caso en tres instituciones de Medellín, Colombia. Gestión y Ambiente, 15(3), 77-87.

Gonzales. D. C. (1996). Principales tendencias y modelos de la Educación Ambiental en el sistema escolar. Revista Iberoamericana de educación, (11), 13-74.

López, N, Fernando. L. (2002). El análisis de contenido como método de investigación. XXI. Revista de educación, (4), 167-180.

Marcote, P. V., \& Suárez, P. A. (2005). Planteamiento de un marco teórico de la Educación Ambiental para un desarrollo sostenible. REEC: Revista electrónica de enseñanza de las ciencias, 4(1), 4.

Mora, W. M. (2011). La Inclusión de la Dimensión Ambiental en la Educación Superior: Un Estudio de Caso en la Facultad de Medio Ambiente de la Universidad Distrital En Bogotá (Doctoral dissertation, Tesis Doctoral Inédita. Universidad de Sevilla (España): Departamento de Didáctica de las Ciencias Experimentales y Sociales. http://fondosdigitales. us. es/tesis/autores/1689).

Pedroza, R. \& Argüello F. (2002). Interdisciplinariedad y Transdisciplinariedad en los Modelos de Enseñanza de la Cuestión Ambiental. Cinta de Moebio. Revista de Epistemología de Ciencias Sociales, (15).

SAUVÉ, Lucie (2004). Una cartografía de corrientes en educación ambiental, En: SATO, Michéle, A pesquisa em educa?áo ambiental: cartografías de urna identidade narrativa em forma^áo, Porto Alegre. Edición digital en: 
Bio - grafia. Escritos sobre la Biología y su Enseñanza. ISSN 2027

Edición Extraordinaria. p.p. 295 - 303

Memorias del VIII Encuentro Nacional de Experiencias en Enseñanza de la Biología y la Educación Ambiental. III Congreso Nacional de Investigación en Enseñanza de la Biología.

http://www.uam.es/departamentos/ciencias/ecologia/documentos\%20descargables/C2 /doc\%202\%20una\%20cartografia\%20de\%20corrientes\%20en\%20EA.pdf

SINA-MINISTERIO DE EDUCACION NACIONAL. Política Nacional Ambiental. Recuperado el 15 de agosto de 2013 en:

http://www.humboldt.org.co/iavh/documentos/politica/politicas_ambientales/2002\%20 Politica\%20Nacional\%20de\%20Educacion\%20Ambiental.pdf

Vidart, D. (1996). Filosofía ambiental: El Ambiente como sistema. Nueva América. Bogotá.

Llorente, M.P., \& Gutiérrez, J. (2006). Modelos teóricos contemporáneos y marcos de fundamentación de la educación ambiental para el desarrollo sostenible. Revista Iberoamericana de Educación, (41), 21-68 